\title{
A CHARACTERIZATION AND A VARIATIONAL INEQUALITY FOR THE MULTIVARIATE NORMAL DISTRIBUTION
}

\author{
WOLFGANG STADJE
}

(Received 2 February 1985; revised 22 May 1986)

Communicated by T. C. Brown

\begin{abstract}
Various generalizations of the Maxwell characterization of the multivariate standard normal distribution are derived. For example the following is proved: If for a $k$-dimensional random vector $X$ there exists an $n \in\{1, \ldots, k-1\}$ such that for each $n$-dimensional linear subspace $H \subset \mathbf{R}^{k}$ the projections of $X$ on $H$ and $H^{\perp}$ are independent, $X$ is normal. If $X$ has a rotationally symmetric density and its projection on some $H$ has a density of the same functional form, $X$ is normal. Finally we give a variational inequality for the multivariate normal distribution which resembles the isoperimetric inequality for the surface measure on the sphere.
\end{abstract}

1980 Mathematics subject classification (Amer. Math. Soc.): $62 \mathrm{H} 05$

\section{Introduction}

This paper is devoted to the consideration of some "geometric" properties of the $k$-dimensional normal distribution $N_{k}\left(\mu, \sigma^{2} I_{k}\right)$, where $k \geqslant 2, \mu \in \mathbb{R}^{k}, \sigma^{2} \geqslant 0$ and $I_{k}$ is the $(k \times k)$-unit matrix. The starting point is the well known Maxwell characterisation stating that $N_{k}\left(0, \sigma^{2} I_{k}\right)$ is the only rotationally symmetric probability measure on $\mathbb{R}^{\kappa}$ for which the coordinates are independent random variables ([6], or [4], pages 160-161; for a related result on random matrices see [7]). Rotational symmetry of a distribution $P$ on $\mathbb{R}^{k}$ is equivalent to the property that the characteristic function (c.f.) $\phi$ (or the density $f$, if it exists) depends only on the euclidean length $|\cdot|$ of its argument. Theorem 1 says that if $|\cdot|$ is replaced by

(C) 1987 Australian Mathematical Society $0263-6115 / 87 \$ A 2.00+0.00$ 
other functions, the classical independence condition characterizes other distributions, for example the Cauchy or the double exponential distribution.

In Theorem 3 the Maxwell characterization is refined in another way. Let $X$ be a $k$-dimensional random vector, $k \geqslant 2$. If there is some $n \in\{1, \ldots, k-1\}$ such that for any $n$-dimensional linear subspace $H$ of $\mathbb{R}^{k}$ and its orthogonal complement $H^{\perp}$ the projected random vectors $p_{H}(X)$ and $p_{H^{\perp}}(X)$ are independent, then $X \sim N_{k}\left(\mu, \sigma^{2} I_{k}\right)$ for some $\mu \in \mathbb{R}^{k}$ and some $\sigma^{2} \geqslant 0$. It is not assumed that $X$ is rotationally symmetric.

Furthermore Section II contains the following result. Let $1 \leqslant n<k$. If $X$ has a density $f(x)=\tilde{f}(|x|), x \in \mathbb{R}^{k}$, and for some $n$-dimensional subspace $H$ of $\mathbb{R}^{k}$ $p_{H}(X)$ has a density $f_{H}$ of the same functional form as that of $X$, that is,

$$
f_{H}(x)=C_{H} \tilde{f}(|x|), \quad x \in H,
$$

( $C_{H}$ some constant), then $X$ is normal (Theorem 2). All three results generalize the Maxwell characterization in different directions. While Theorem 1 integrates the classical statement into a whole class of characterizations, in the other two theorems the restriction to one-dimensional projections is dropped. In Section III we exhibit an interesting relation between the standard normal distribution $N:=N_{k}\left(0, \sigma^{2} I_{k}\right)$ and the geometry of euclidean space indicating that $N$ plays a similar role for $\mathbb{R}^{k}$ as the surface measure for the unit sphere $S^{k-1}$. The result is expressed by a "variational inequality" resembling the isoperimetric property of $S^{k-1}$. This property can be expressed in the following way: Among all compact subsets $K$ of $S^{k-1}$ for which the normalized surface measure $\mu_{k}(K)$ is equal to some fixed $c \in[0,1]$ the geodesic balls have minimal Minkowski surface. Here the Minkowski surface $O(A)$ of a Borel measurable set $A \subset S^{k-1}$ is defined by

$$
O(A):=\liminf _{\delta \rightarrow 0}\left(\mu_{k}\left(U_{k, \delta}^{\Delta}(A)-\mu_{k}(A)\right)\right) / \delta,
$$

where $U_{k, \delta}^{\Delta}(A)$ denotes the closed geodesic $\delta$-neighbourhood of $A$ with respect to $S^{k-1}$. The isoperimetric property is a corollary of the following fundamental relation: If $H$ is a geodesic ball and $K$ is compact, then for all $\delta>0$

$$
\mu_{k}(H) \leqslant \mu_{k}(K) \Rightarrow \mu_{k}\left(U_{k, \delta}^{\Delta}(H)\right) \leqslant \mu_{k}\left(U_{k, \delta}^{\Delta}(K)\right) .
$$

The first proof of (2) was given in [8], it has then been simplified in [1]. A very short and elegant more recent proof can be found in [2].

The implication (2) will be seen to hold for $N$ in an analogous form. If $\mu_{k}$ is replaced by $N$, the geodesic neighbourhoods by euclidean ones and $H$ now denotes a half space in $\mathbb{R}^{k}$, then (2) remains valid. It is not known (to the author), whether $N$ is the only probability measure on $\mathbb{R}^{k}$ with this "isoperimetric" property. This would yield a new type of characterization of $N$ quite different from the classical ones. The result is derived by projection techniques similar to those applied in Section II. 


\section{A characterization of $N\left(\mu, \sigma^{2} I_{k}\right)$}

Let $k \geqslant 2$. Our first theorem generalizes the following result: If $X=$ $\left(X_{1}, \ldots, X_{k}\right)^{\prime}$ has a characteristic function of the form $F(|x|)$ and independent components, then $X \sim N\left(0, \sigma^{2} I_{k}\right)$. The role of $|\cdot|$ will be played by an arbitrary function of the type $h\left(\left|x_{1}\right|\right)+\cdots+h\left(\left|x_{k}\right|\right)$, where $h:[0, \infty) \rightarrow[0, \infty)$ is continuous, strictly increasing and $h(0)=0$.

TheOrEm 1. Let $h:[0, \infty) \rightarrow[0, \infty)$ be continuous and bijective and let the $k$-dimensional random vector $X=\left(X_{1}, \ldots, X_{k}\right)^{\prime}$ have a density (or characteristic function) $f$ of the form

$$
f(x)=F\left(h\left(\left|x_{1}\right|\right)+\cdots+h\left(\left|x_{k}\right|\right)\right), \quad x=\left(x_{1}, \ldots, x_{k}\right)^{\prime} \in \mathbb{R}^{k},
$$

for some measurable function $F:[0, \infty) \rightarrow \mathbb{C}$. Then $X_{1}, \ldots, X_{k}$ are independent, if and only if

for some $\alpha, \beta \geqslant 0$.

$$
f(x)=\beta \exp \left\{-\alpha \sum_{i=1}^{k} h\left(\left|x_{i}\right|\right)\right\}
$$

Proof. Let $f_{1}, \ldots, f_{k}$ be the densities (resp. characteristic functions) of $X_{1}, \ldots, X_{k}$ and $\tilde{f}:=F \circ h$. The independence of $X_{1}, \ldots, X_{k}$ is equivalent to

$$
f_{1}\left(x_{1}\right) \cdots f_{k}\left(x_{k}\right)=\tilde{f}\left(h^{-1}\left(\sum_{i=1}^{k} h\left(\left|x_{i}\right|\right)\right)\right) .
$$

If $f_{i}(0)=0$ for some $i,(5)$ and the conditions on $h$ imply $\tilde{f} \equiv 0$ and thus $f \equiv 0$ which is impossible. So we obtain by taking $x_{j}=0$ for all $j \neq i$ in (5)

$$
f_{i}\left(x_{i}\right)=c_{i} \tilde{f}\left(\left|x_{i}\right|\right), \quad i=1, \ldots, k,
$$

for some constants $c_{1}, \ldots, c_{k}$. Inserting (6) into (5) yields

$$
\tilde{f}\left(\left|x_{1}\right|\right) \cdots \tilde{f}\left(\left|x_{k}\right|\right)=C \tilde{f}\left(h^{-1}\left(\sum_{i=1}^{k} h\left(\left|x_{i}\right|\right)\right)\right) .
$$

If we take $u_{i}=h\left(\left|x_{i}\right|\right)$, we arrive at

$$
F\left(u_{1}\right) \cdots F\left(u_{k}\right)=C F\left(u_{1}+\cdots+u_{k}\right), \quad u_{1}, \ldots, u_{k} \in[0, \infty) .
$$

The only measurable solution of (8) is given by $F(u)=\beta e^{-\alpha u}$, where $\beta=C^{1 /(k-1)}$ and $\alpha$ is a complex constant. If $f$ is a characteristic function, it follows from $f(0)=1$ that $\beta=1$. Since $f(x)=f(-x), f$ is real-valued, and from $|f| \leqslant 1$ we can conclude that $\alpha \geqslant 0$. If $f$ is a density, $\alpha, \beta>0$ follows from $f \geqslant 0$ and $\int f d x=1$.

ExAmples. (i) Let $h(t):=|t|^{p}$ for some $p>0$. Then $f(x)=$ $\beta \exp \left\{-\alpha \sum_{i=1}^{k}\left|x_{i}\right|^{p}\right\}$. The normal case comes out for $p=2$.

(ii) Let $h(t):=\log \left(1+|t|^{p}\right), p>0$. Then we get $f(x)=\beta\left[\Pi_{i=1}^{k}\left(1+\left|x_{i}\right|^{p}\right)\right]^{-a}$. The Cauchy distribution belongs to this class. 
We now prove two theorems on $N_{k}\left(\mu, \sigma^{2} I_{k}\right)$ which generalize the Maxwell characterization in different ways. The following notation is used: For $n \in \mathbb{N}, \lambda^{n}$ is the $n$-dimensional Lebesgue measure, $\mathfrak{B}^{n}$ is the Borel $\sigma$-algebra in $\mathbb{R}^{n} . p_{H}$ denotes the projection on the linear subspace $H$ of $\mathbb{R}^{k}, H^{\perp}$ the orthogonal complement of $H$ in $\mathbf{R}^{k}$. For a probability measure $P$ on $\left(R^{k}, \mathfrak{B}^{k}\right) P_{H}$ is the projection $P p_{H}^{-1}$ of $P$.

THEOREM 2. Let $P$ be a $k$-dimensional rotationally symmetric distribution with density $f(x)=\tilde{f}(|x|)$. If a projection $P_{H}$ of $P$ on some lower-dimensional linear subspace $H$ has a density $f_{H}$ of the same functional form as $P$, that is, $f_{H}(x)=$ $C \tilde{f}(|x|)$ for some normalizing constant $C$, then $P$ is normal.

This result is somewhat surprising, if one notes that for all rotationally symmetric $P$ the characteristic function $\phi_{H}$ of $P_{H}$ is of the same functional form as that of $P\left(\phi_{H}(\lambda)=\tilde{\phi}(|\lambda|)\right.$, where $\zeta \rightarrow \tilde{\phi}(|\zeta|), \zeta \in \mathbb{R}^{k}$, is the characteristic function of $P$ ).

Proof of Theorem 2. We can assume that $P$ has a continuous density $f$, since otherwise we can consider the convolution $P * N_{k}\left(0, I_{k}\right)$ and then use the proof below to show that $P * N_{k}\left(0, I_{k}\right)=N_{k}\left(0, \sigma^{2} I_{k}\right)$ for some $\sigma^{2} \geqslant 0$ which implies that $\sigma^{2} \geqslant 1$ and $P=N_{k}\left(0,\left(\sigma^{2}-1\right) I_{k}\right)$. Thus let $f$ and, consequently, also $\tilde{f}$ be continuous. Let $H_{n}:=\left\{x \in \mathbb{R}^{k} \mid x_{j}=0, j>n\right\} . p_{H_{n}}(X)$ has the continuous density $f_{H_{n}}$ given by

$$
f_{H_{n}}(x)=\frac{2 \pi^{(k-n) / 2}}{\Gamma((k-n) / 2)} \int_{|x|}^{\infty} c\left(c^{2}-|x|^{2}\right)^{(k-n-2) / 2} \tilde{f}(c) d c
$$

(see the remark following this proof). There are continuous functions $\tilde{f}_{H_{n}}, \tilde{f}_{H_{n}^{\perp}}$ : $[0, \infty) \rightarrow[0, \infty)$ such that $f_{H_{n}}(x)=\tilde{f}_{H_{n}}(|x|), f_{H_{n}^{\perp}}(x)=\tilde{f}_{H_{n}}(|x|)$ for all $x \in \mathbb{R}^{k}$. Now for $t>0$ we have $\tilde{f}(t)=\tilde{f}_{H_{n}}(0) \tilde{f}_{H_{n}}(t)$. Further, if we set $m:=\tilde{f}_{H_{n}}(0)$, we obtain

$$
\begin{aligned}
\tilde{f}(t) & =m \tilde{f}_{H_{n}^{\perp}}(t)=m \int_{\mathbf{R}^{n}} \tilde{f}\left(\sqrt{t^{2}+|x|^{2}}\right) \lambda^{n}(d x) \\
& =m^{2} \int_{\mathbf{R}^{n}} \tilde{f}_{H_{n}^{\perp}}\left(\sqrt{t^{2}+|x|^{2}}\right) \lambda^{n}(d x) \\
& =m^{2} \int_{\mathbf{R}^{n}} \int_{\mathbf{R}^{n}} \tilde{f}\left(\sqrt{t^{2}+\left|x^{(1)}\right|^{2}+\left|x^{(2)}\right|^{2}}\right) \lambda^{n}\left(d x^{(1)}\right) \lambda^{n}\left(d x^{(2)}\right) \\
& =m^{2} \int_{\mathbf{R}^{2 n}} \tilde{f}\left(\sqrt{t^{2}+|x|^{2}}\right) \lambda^{2 n}(d x) \\
& =\cdots=m^{j} \int_{\mathbf{R}^{\prime n}} \tilde{f}\left(\sqrt{t^{2}+|x|^{2}}\right) \lambda^{j n}(d x)
\end{aligned}
$$


for all $j \in \mathbb{N}$. Let $Q_{j}$ be the probability measure on $\mathbb{R}^{(j+1) n}$ with density $y \rightarrow C_{j} \tilde{f}(|y|), y \in \mathbb{R}^{(j+1) n}$, where $C_{j}$ is some normalizing constant. By (9), $P$ can be expressed as a projection of $Q_{j}$. Since this property of $P$ holds for arbitrarily large $j$, it follows from Theorem 2 of [3], p. 394, that $P$ is a variance mixture of the normal distributions $N_{k}\left(0, \sigma^{2} I_{k}\right)$. Hence $f$ can be written as

$$
f(x)=\int_{0}^{\infty}(2 \pi)^{-k / 2} \sigma^{-k} e^{-|x|^{2} / 2 \sigma^{2}} \alpha(d \sigma), \quad x \in \mathbb{R}^{k},
$$

for some probability measure $\alpha$ on $(0, \infty)$.

Inserting (10) into the first line of (9) one obtains

$$
\begin{aligned}
\int_{0}^{\infty}(2 \pi)^{-k / 2} \sigma^{-k} e^{-|x|^{2} / 2 \sigma^{2}} \alpha(d \sigma) & \\
& =m \int_{0}^{\infty} \int_{\mathbf{R}^{n}}(2 \pi)^{-k / 2} \sigma^{-k} e^{-\left(|x|^{2}+|y|^{2}\right\} / 2 \sigma^{2}} \lambda^{n}(d y) \alpha(d \sigma) \\
& =\int_{0}^{\infty} m(2 \pi)^{(n-k) / 2} \sigma^{n-k} e^{-|x|^{2} / 2 \sigma^{2}} \alpha(d \sigma) .
\end{aligned}
$$

Next we have to note that the mapping $f \rightarrow \alpha$ is one-to-one: If one defines the probability measure $\bar{\alpha}(0, t]:=\alpha(0, \sqrt{t}]$, the Laplace transform of $\bar{\alpha}$ coincides with the characteristic function $\phi$ of $f$, since

$$
\phi(u)=\int_{0}^{\infty} e^{-|u|^{2} \sigma^{2} / 2} \alpha(d \sigma)=\int_{0}^{\infty} e^{-|u|^{2} \sigma / 2} \bar{\alpha}(d \sigma) .
$$

But $\alpha$ is determined by $\bar{\alpha}$, and $\bar{\alpha}$ by its Laplace transform which can be computed from $f$.

Hence the measures $\alpha(d \sigma)$ and $m(2 \pi)^{n / 2} \sigma^{n} \alpha(d \sigma)$ are equal which is possible only if $\alpha$ is the point mass in $(2 \pi)^{-1 / 2} m^{-1 / n}$. By (10) it is now seen that

$$
f(x)=m^{k / n} e^{-|x|^{2} m^{2 / n} \pi}
$$

The proof is now complete.

REMARK. Equation (8) follows easily from the results of Eaton [3]. He proved that if $X$ is rotationally symmetric, then $X /|X|$ and $|X|$ are independent, and $p_{H_{n}}(X /|X|)$ has the density

$$
g_{k, n}(u)=\frac{\Gamma\left(\frac{k}{2}\right)}{\pi^{n / 2} \Gamma((k-n) / 2)}\left(1-|u|^{2}\right)^{(k-n-2) / 2} 1_{(-1,1)}(|u|)
$$

for $u \in H_{n}$. Hence,

$$
\begin{aligned}
P\left(p_{H_{n}}(X) \leqslant t\right) & =\int P\left(p_{H_{n}}(X) \leqslant t|| X \mid=c\right) P(|X| \in d c) \\
& =\int P\left(p_{H_{n}}(X /|X|) \leqslant \frac{t}{c}\right) \nu(d c),
\end{aligned}
$$


where $t \in \mathbb{R}^{k}$ and $\nu(a, b]:=P\{x|| x \mid \in(a, b]\}, a, b \in \mathbb{R}$. Inserting (15) into (14) shows that $p_{H_{n}}(X)$ has the density

$$
f_{H_{n}}(x)=\frac{\Gamma(k / 2)}{\pi^{n / 2} \Gamma((k-n) / 2)} \int_{|x|}^{\infty} c^{-n}\left(1-\frac{|x|^{2}}{c^{2}}\right)^{(k-n-2) / 2} \nu(d c) .
$$

(8) now follows, because $\nu$ has the density

$$
\left(2 \pi^{k / 2} / \Gamma(k / 2)\right) c^{k-1} \tilde{f}(c) 1_{(0, \infty)}(c), \quad c \in \mathbb{R} .
$$

Formula (15) incidentally shows that $f_{H_{n}}$ is non-increasing, if $k-n \geqslant 2$, as has been shown in [3], Proposition 1. However this is not true for $k-n=1$ (a case not excluded in [3]), as can be seen by the example of the uniform distribution on $S^{1}$. Then

$$
f_{H_{1}}(u)=1 / \pi\left(1-u^{2}\right)^{1 / 2}, \quad-1<u<1 .
$$

The final theorem of this section shows that a "Maxwell characterization" of $N_{k}\left(\mu, \sigma^{2} I_{k}\right)$ is possible without the assumption of rotational symmetry.

THEOREM 3. Let $X$ be a $k$-dimensional random vector for which there exists a $n \in\{1, \ldots, k-1\}$ such that $p_{H}(X)$ and $p_{H^{+}}(X)$ are independent for each $n$ dimensional linear subspace $H$ of $\mathbb{R}^{k}$. Then $X \sim N_{k}\left(\mu, \sigma^{2} I_{k}\right)$ for some $\mu \in \mathbb{R}^{k}$ and $\sigma^{2} \geqslant 0$.

Proof. Let $P$ be the distribution of $X$. We have to show that $P$ is rotationally symmetric, for then Theorem 2 can be applied.

If the result holds for all symmetric probability measures $Q$ (satisfying $Q(B)=$ $Q(-B)$ for all $\left.B \in B^{k}\right)$, it is true in general. For if we define $\bar{P}(B):=P(-B)$, $B \in \mathfrak{B}^{k}$, the convolution $\tilde{P}=P * \bar{P}$ of $P$ and $\bar{P}$ clearly fulfils the assumptions of the theorem and is symmetric so that we have $\tilde{P}=N_{k}\left(0, \sigma^{2} I_{k}\right)$ for some $\sigma \in[0, \infty)$. Then the multivariate version of Cramèr's theorem ([9], page 46) implies that $P=N_{k}\left(\mu, \frac{1}{2} \sigma^{2} I_{k}\right)$ for some $\mu \in \mathbb{R}^{k}$.

Hence we can assume that $P$ is symmetric. We show that in this case $P$ is even rotationally symmetric. It suffices to prove that, if $\lambda, \tilde{\lambda} \in \mathbb{R}^{k}$ satisfy $|\lambda|=|\tilde{\lambda}|$, then $\phi(\lambda)=\phi(\tilde{\lambda})$, where $\phi$ is the characteristic function of $P$. We can choose an orthonormal basis $e^{1}, \ldots, e^{k}$ of $\mathbb{R}^{k}$ such that $\lambda=\left(\lambda_{1}, \lambda_{2}, 0, \ldots, 0\right)^{\prime}$ and $\tilde{\lambda}=$ $\left(-\lambda_{1}, \lambda_{2}, 0, \ldots, 0\right)^{\prime}$ are the coordinate representations of $\lambda$ and $\tilde{\lambda}$ with respect to this basis. Let $H$ be the span of $e^{2}, \ldots, e^{n+1}$. Then clearly $p_{H}(\lambda)=$ $\left(0, \lambda_{2}, 0, \ldots, 0\right)^{\prime}=p_{H}(\tilde{\lambda})$ and $p_{H^{\perp}}(\lambda)=\left(\lambda_{1}, 0, \ldots, 0\right)^{\prime}=-p_{H^{\perp}}(\tilde{\lambda})$. Now it follows from the assumptions that

$$
\begin{aligned}
\phi(\lambda) & =\phi\left(p_{H}(\lambda)\right) \phi\left(p_{H^{\perp}}(\lambda)\right)=\phi\left(p_{H}(\tilde{\lambda})\right) \phi\left(-p_{H^{\perp}}(\tilde{\lambda})\right) \\
& =\phi\left(p_{H}(\tilde{\lambda})\right) \phi\left(p_{H^{\perp}}(\tilde{\lambda})\right)=\phi(\tilde{\lambda}) .
\end{aligned}
$$




\section{A variational inequality for $N$}

Our final aim is to prove the inequality for $N$ announced in the introduction: Among all Borel sets $B$ of given $N$-measure the half spaces minimize the $N$-measure of their euclidean $\varepsilon$-neighbourhood for arbitrary $\varepsilon>0$. This result indicates a similarity between the relation of $S^{k-1}$ to its surface measure and that of $\mathbb{R}^{k}$ to the standard normal distribution.

Let $\mu_{k}$ be the normalized surface area of $S^{k-1}$. In the following $\mu_{k}$ is considered as a measure on $\left(\mathbb{R}^{k}, \mathfrak{B}^{k}\right)$ concentrated on $S^{k-1}$ by setting $\mu_{k}(B):=\mu_{k}\left(B \cap S^{k-1}\right)$. We first note that the conclusion (2) in Section I remains valid if $K$ is replaced by an arbitrary $B \in B^{k}$, because for all $\varepsilon^{\prime}<\varepsilon$

$$
\begin{aligned}
\mu_{k}\left(U_{k, \varepsilon^{\prime}}^{\Delta}(H)\right) & \leqslant \mu_{k}\left\{y \in \mathbb{R}^{k}|\exists x \in \mathrm{cl}(B):| x-y \mid<\varepsilon\right\} \\
& =\mu_{k}\left\{y \in \mathbb{R}^{k}|\exists x \in B:| x-y \mid<\varepsilon\right\} \\
& \leqslant \mu_{k}\left\{U_{k, \varepsilon}^{\Delta}\left(B \cap S^{k-1}\right)\right\} .
\end{aligned}
$$

Let $\varepsilon^{\prime} \uparrow \varepsilon$.

Theorem 3. Let $B \in \mathfrak{B}^{k}$ and $H=\left\{x \in \mathbb{R}^{k} \mid x^{\prime} x^{0} \leqslant a\right\}, a \in \mathbb{R}, x^{0} \in \mathbb{R}^{k}$. Then we have

$$
N(H) \leqslant N(B) \Rightarrow N\left(H^{\varepsilon}\right) \leqslant N\left(B^{\varepsilon}\right) \text { for all } \varepsilon>0 .
$$

Proof. By $p_{n k}: \mathbb{R}^{n} \rightarrow \mathbb{R}^{k}$ we denote the projection $\left(x_{1}, \ldots, x_{n}\right)^{\prime} \rightarrow$ $\left(x_{1}, \ldots, x_{k}\right)^{\prime}, n>k$. Let $\mu_{n, c}$ be the uniform distribution on $c S^{k-1}:=\{x \in$ $\left.\mathbb{R}^{k}|| x \mid=c\right\}$ (also considered as a measure on $\mathbb{R}^{k}$ ). By (18) we immediately obtain

$$
\mu_{n, \sqrt{n}} p_{n k}^{-1} \rightarrow N \text { in total variation, as } n \rightarrow \infty,
$$

because the corresponding sequence of densities converges pointwise to the density of $N$. Without loss of generality we assume $x^{0}=(1,0, \ldots, 0)^{\prime}$ and consequently $H=\left\{x \in \mathbb{R}^{k} \mid x_{1} \leqslant a\right\}$. Let $\Delta_{n, c}$ be the geodesic metric on $c S^{n-1}$ and $U_{n, c, \varepsilon}^{\Delta}(B)$ be the closed $\varepsilon$-neighbourhood with respect to $\Delta_{n, c}$. Then it is seen that

$$
U_{n, \sqrt{n}, \ell}^{\Delta}\left(\sqrt{n} S^{n-1} \cap p_{n k}^{-1}(H)\right)=p_{n k}^{-1}\left(\left\{x \in \mathbb{R}^{k} \mid x_{1} \leqslant a+\varepsilon_{n}\right\}\right) \cap \sqrt{n} S^{n-1},
$$

where $\varepsilon$ and $\varepsilon_{n}$ satisfy the equation

$$
\varepsilon=\sqrt{n}\left(\arccos \left(\frac{a}{\sqrt{n}}\right)-\arccos \left(\frac{a+\varepsilon_{n}}{\sqrt{n}}\right)\right) .
$$


The Taylor expansion of arccos yields

$$
\varepsilon=\left(1-\xi_{n}^{2}\right)^{-1 / 2} \varepsilon_{n} \quad \text { for some } \xi_{n} \in\left[a / \sqrt{n},\left(a+\varepsilon_{n}\right) / \sqrt{n}\right] .
$$

From this it is seen that $\lim _{n \rightarrow \infty} \varepsilon_{n}=\varepsilon$. By (22) for each $\delta>0$ there exists a $n_{0} \in \mathbb{N}$ such that for $n \geqslant n_{0}$

$$
\begin{aligned}
\mu_{n, \sqrt{n}} & \left(U_{n, \sqrt{n}, \varepsilon}^{\Delta}\left(\sqrt{n} S^{n-1} \cap p_{n k}^{-1}(H)\right)\right) \\
& =\mu_{n, \sqrt{n}}\left(p_{n k}^{-1}\left(\left\{x \in \mathbb{R}^{k} \mid x_{1} \leqslant a+\varepsilon_{n}\right\}\right)\right) \\
& \in\left[N\left\{x \in \mathbb{R}^{k} \mid x_{1} \leqslant a+\varepsilon-\delta\right\}, N\left\{x \in \mathbb{R}^{k} \mid x_{1} \leqslant a+\varepsilon+\delta\right\}\right] .
\end{aligned}
$$

From (25) it follows that

$$
\lim _{n \rightarrow \infty} \mu_{n, \sqrt{n}}\left(U_{n, \sqrt{n}, \varepsilon}^{\Delta}\left(\sqrt{n} S^{n-1} \cap p_{n k}^{-1}(H)\right)\right)=N\left(H^{\varepsilon}\right) .
$$

Further from $|x-y| \leqslant \Delta_{n, \sqrt{n}}(x, y)$ for all $x, y \in \sqrt{n} S^{n-1}$ we can conclude that

$$
U_{n, \sqrt{n}, \varepsilon}^{\Delta}\left(p_{n k}^{-1}(B)\right) \subset p_{n k}^{-1}\left(B^{\varepsilon}\right) \cap \sqrt{n} S^{n-1} .
$$

Set $H_{b}:=\left\{x \in \mathbb{R}^{k} \mid x_{1} \leqslant b\right\}$. Under the supposition of (21) (22) shows that for each $b<a$ there is a $n_{1} \in \mathbb{N}$ so that

$$
\mu_{n, \sqrt{n}}\left(p_{n k}^{-1}\left(H_{b}\right)\right) \leqslant \mu_{n, \sqrt{n}}\left(p_{n k}^{-1}(B)\right) \quad \text { for all } n \geqslant n_{1} .
$$

The isoperimetric inequality (2), when applied to $\sqrt{n} S^{n-1}$, yields for all $\varepsilon>0$ and $n \geqslant n_{1}$

$$
\mu_{n, \sqrt{n}}\left(U_{n, \sqrt{n}, \varepsilon}^{\Delta}\left(p_{n k}^{-1}\left(H_{b}\right) \cap \sqrt{n} S^{n-1}\right)\right) \leqslant \mu_{n, \sqrt{n}}\left(U_{n, \sqrt{n}, \varepsilon}^{\Delta}\left(p_{n k}^{-1}(B) \cap \sqrt{n} S^{n-1}\right)\right) .
$$

Further we have (again by (22))

$$
\lim _{n \rightarrow \infty} \mu_{n, \sqrt{n}}\left(p_{n k}^{-1}\left(B^{\varepsilon}\right)\right)=N\left(B^{\varepsilon}\right) .
$$

Finally combining (26) (applied to $H_{b}$ ), (24), (27) and (30) we obtain

$$
\begin{aligned}
N\left(H_{b}^{\varepsilon}\right) & =\lim _{n \rightarrow \infty} \mu_{n, \sqrt{n}}\left(U_{n, \sqrt{n}, \varepsilon}^{\Delta}\left(p_{n k}^{-1}\left(H_{b}\right) \cap \sqrt{n} S^{n-1}\right)\right) \\
& \leqslant \liminf _{n \rightarrow \infty} \mu_{n, \sqrt{n}}\left(U_{n, \sqrt{n}, \varepsilon}^{\Delta}\left(p_{n k}^{-1}(B) \cap \sqrt{n} S^{n-1}\right)\right) \\
& \leqslant \liminf _{n \rightarrow \infty} \mu_{n, \sqrt{n}}\left(p_{n k}^{-1}\left(B^{\varepsilon}\right)\right)=N\left(B^{\varepsilon}\right) .
\end{aligned}
$$

$b \uparrow a$ yields the conclusion of (21).

Whether $N$ is the only distribution on $\mathbb{R}^{k}$ which satisfies (21) is an open question. 


\section{References}

[1] A. Dinghas, 'Einfacher Beweis der isoperimetrischen Eigenschaft der Kugel in Riemannschen Räumen konstanter Krümmung', Math. Nachr. 2 (1949), 148-162.

[2] T. Figiel, J. Lindenstrauss and V. D. Milman, 'The dimension of almost spherical sections of convex bodies', Acta Math. 139 (1977), 53-94.

[3] M. L. Eaton, 'On the projections of isotropic distributions', Ann. Statist. 9 (1981), 391-400.

[4] M. Fisz, Probability theory and mathematical statistics, (Wiley, New York-London, 1963).

[5] J. Lord, 'The use of Hankel transforms in statistics I', Biometrika 41 (1954), 44-55.

[6] A. M. Mathai and G. Pederzoli, Characterizations of the normal probability law, (Wiley Eastern Limited, New Delhi-Bangalore-Bombay, 1977).

[7] W. H. Olson and V. R. Rao Uppuluri, 'Characterization of the distribution of a random matrix by rotational invariance', Sankhy $\bar{a} A$ 32 (1970), 325-328.

[8] E. Schmidt, 'Die Brunn-Minkowski-Ungleichung', Math. Nachr. 1 (1948), 81-157.

[9] M. S. Srivastava and C. G. Khatri, An introduction to multicuriate statistics, (North-Holland. New York-Oxford, 1979).

\section{Fachbereich Mathematik}

Universität Osnabrück

Albrechtstrasse 28

West Germany 